\title{
Direct Observation of Correlated Interdomain Motion in Alcohol Dehydrogenase
}

\author{
Ralf Biehl, ${ }^{1}$ Bernd Hoffmann, ${ }^{2}$ Michael Monkenbusch, ${ }^{1}$ Peter Falus, ${ }^{3}$ Sylvain Préost,${ }^{4}$ Rudolf Merkel, ${ }^{2}$ and Dieter Richter ${ }^{1}$ \\ ${ }^{1}$ Institut für Festkörperforschung, Forschungszentrum Jülich, D-52425 Jülich, Germany \\ ${ }^{2}$ Institut für Bio- und Nanosysteme, Forschungszentrum Jülich, D-52425 Jülich, Germany \\ ${ }^{3}$ Institut Laue Langevin, F-38042 Grenoble, France \\ ${ }^{4}$ Hahn Meitner Institut, D-14109 Berlin, Germany \\ (Received 9 April 2008; published 26 September 2008)
}

\begin{abstract}
Interdomain motions in proteins are essential to enable or promote biochemical function. Neutron spinecho spectroscopy is used to directly observe the domain dynamics of the protein alcohol dehydrogenase. The collective motion of domains as revealed by their coherent form factor relates to the cleft opening dynamics between the binding and the catalytic domains enabling binding and release of the functional important cofactor. The cleft opening mode hardens as a result of an overall stiffening of the domain complex due to the binding of the cofactor.
\end{abstract}

DOI: 10.1103/PhysRevLett.101.138102

PACS numbers: 87.15.H-, 87.14.E-, 87.64.Bx

Large scale motions of protein domains are critical for protein function as nanomachines. Understanding the underlying processes requires a simultaneous access to the proper length and time scales close to natural environmental conditions in solution. This is realized by a combination of small angle neutron scattering (SANS) providing structural characterization and neutron spin echo spectroscopy (NSE) furnishing space time resolution on $\mathrm{nm}$ and $\mathrm{ns}$ scales. We investigate here the protein alcohol dehydrogenase $(\mathrm{ADH})$ responsible for the interconversion between alcohol and ketones. In addressing the coherent dynamic form factors the experiments demonstrate a novel access towards the large scale cooperative dynamics of protein domains with its high relevance for biological function.

Functionally, the studied ADH from Saccharomyces cerevisiae is part of the large family of zinc containing alcohol dehydrogenases. They convert ethanol to acetaldehyde, a reaction that can be catalyzed in both directions. In vivo as well as in vitro systems using the catalytic conversion of ethanol to acetaldehyde depends on nicotinamide adenine dinucleotide $\left(\mathrm{NAD}^{+}\right)$as the cofactor. Such catalysis is essential for many organisms in order to use ethanol as a carbon source as well as detoxification reaction at high alcohol concentrations.

Structurally ADH can function as a dimer [1] or a tetramer as found in S. cerevisiae [2]. Crystal structure analysis reveals for each subunit a dumbbell like structure with two globular domains: the catalytic domain containing the two zinc atoms and the cofactor binding domain [3] [Fig. 1(b)]. The variable cleft between both domains contains the active site of ADH [4], where the cofactor is bound for the catalytic process.

We studied the large scale thermal protein fluctuations on the length scale of the domains and simultaneously on the time scale of their motions. Applying a normal mode analysis the observed dynamics may be understood in terms of a cleft opening mode assisting the incorporation of the functionally important NAD cofactor.
ADH from S. cerevisiae (PDB code 2hcy) was purchased (Sigma) and analyzed for purity $(95 \%)$ by SDSpage electrophoresis. ADH was resuspended in $\mathrm{D}_{2} \mathrm{O}$ buffer $\left(10 \mathrm{mM} \mathrm{NaPO}_{4}, 100 \mathrm{mM} \mathrm{NaCl}, p \mathrm{H} \mathrm{7.5)}\right.$ ) at concentrations from $1 \% \mathrm{wt} / \mathrm{vol}$ to $6 \% \mathrm{wt} / \mathrm{vol}$. For ADH saturation with the cofactor a twofold molar concentration of $\mathrm{NAD}^{+}$was added to the protein. Samples without the cofactor were depleted for $\mathrm{NAD}^{+}$by addition of ethanol in a twofold molar ratio. For subsequent experiments differential scanning calorimetric analyses showed no change demonstrating structural integrity of proteins and equal ADH activity

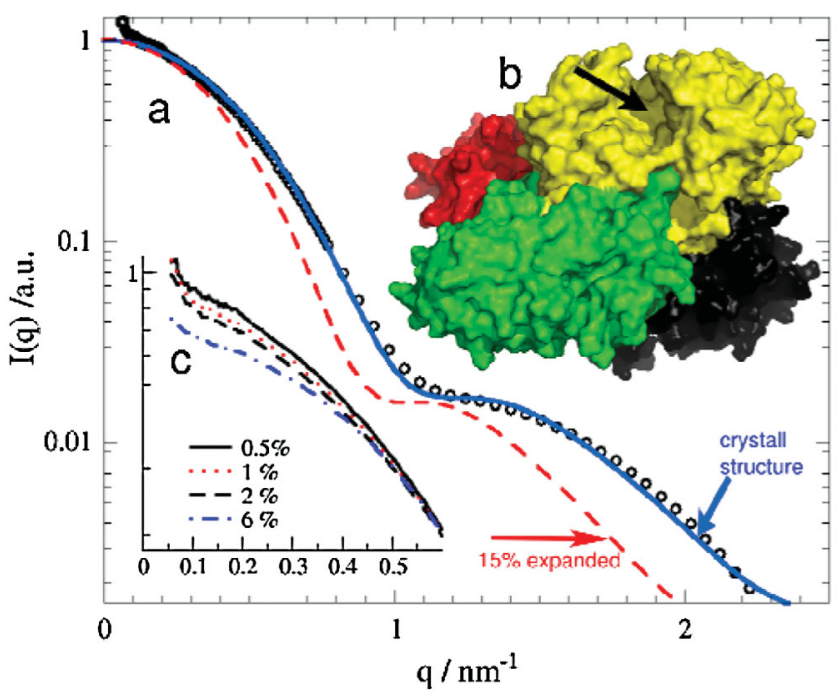

FIG. 1 (color). (a) SANS intensity from ADH with bound cofactor at $0.5 \% \mathrm{wt} / \mathrm{vol}$ conc. (circles) and protein form factors (lines see text). The shoulder around $1 \mathrm{~nm}^{-1}$ is characteristic for the tetrameric structure. (b) Illustration of the overall protein structure with two crossed dimers (green/yellow in front, red/ black behind). The yellow monomer shows the cofactor binding cleft (arrow) separating the inner cofactor binding domain and the exterior catalytic domain. (c) Concentration scaled, low $q$ scattering intensity of the protein solution. 
was found before and after SANS measurements (enzymatic assay according to Kägi and Vallee [5]). Monodispersity was verified by dynamic light scattering (DLS).

The solution structure was explored by SANS experiments (Instrument V4, HMI, Berlin) in $\mathrm{D}_{2} \mathrm{O}$ buffer solutions at different concentrations. The wavelength was $0.6 \mathrm{~nm}$ and the temperature was $5{ }^{\circ} \mathrm{C}$. Figure 1 (a) presents the resulting corrected scattering intensities $I(q)$ as the function of the scattering vector $q=4 \pi / \lambda(\sin \theta / 2)(\lambda$ : neutron wavelength, $\theta$ : scattering angle). The coherent scattering intensity in a solution of equal particles is given by $I(q) \propto N S(q) F(q)$ where the structure factor $S(q)$ results from the interparticle interactions of $N$ scatterers and depends on concentration, while the concentration independent form factor

$$
F(q)=\sum_{j, k}\left\langle b_{j} b_{k} \exp \left(i \mathbf{q}\left(\mathbf{r}_{\mathbf{j}}-\mathbf{r}_{\mathbf{k}}\right)\right)\right\rangle
$$

describes the scattering of the single tetramer. $b_{j}$ are the atomic scattering lengths and $\mathbf{r}_{\mathbf{j}}$ the atomic position vectors. For the form factor calculation the coordinates of the crystal structure from the protein data bank are used and hydrogen exchange is considered according to methods described by Jacrot [6]. All calculations were done with the procedures assembled in the molecular modelling tool kit MMTK [7]. As may be seen from Fig. 1(a) the structural model based on the crystal structure (solid line) is in very good agreement with the SANS results. Figure 1(c) presents the scattering intensities after scaling with concentration. The observed decrease of the scattering intensity at low $q$ is caused by the structure factor $S(q)$, which is extracted in dividing by the lowest concentration data.

The translational diffusion coefficient $D_{\text {trans }}$ was determined by DLS at various concentrations and temperatures from $4{ }^{\circ} \mathrm{C}$ to $10^{\circ} \mathrm{C}$ with and without the bound cofactor. The temperature independent hydrodynamic radius $R_{\mathrm{H}}=$ $4.5 \pm 0.2 \mathrm{~nm}$ compares well with the radius of gyration $R_{\mathrm{G}}=3.4 \mathrm{~nm}$ of the crystal structure, assuming $R_{\mathrm{G}} \approx$ $\sqrt{3 / 5} R_{\mathrm{H}}=3.5 \mathrm{~nm}$ for a sphere. $D_{\text {trans }}$ of $23.5 \pm$ $2 \mu \mathrm{m}^{2} / \mathrm{s}\left(T=5^{\circ} \mathrm{C}\right)$ was measured independent of protein concentration.

The dynamics on the scale of the protein was investigated with NSE revealing the intermediate dynamic structure factor $I(q, t) / I(q)$ in a time range from about 100 ps to 170 ns. NSE experiments were done at the IN15 at ILL, Grenoble at wavelengths from $0.6 \mathrm{~nm}$ up to $1.5 \mathrm{~nm}$ in a temperature controlled environment at $5{ }^{\circ} \mathrm{C}$. The concentration was $1 \% \mathrm{wt} / \mathrm{vol}$ without and $5 \% \mathrm{wt} / \mathrm{vol}$ with and without the bound cofactor. Figure 2 displays NSE results in a logarithmic fashion for a larger number of $q$ 's. In each case single exponential fits are included demonstrating that the dynamic structure factor may be reasonably well described in terms of a single exponential decay. From the spectra best estimates for their first cumulant

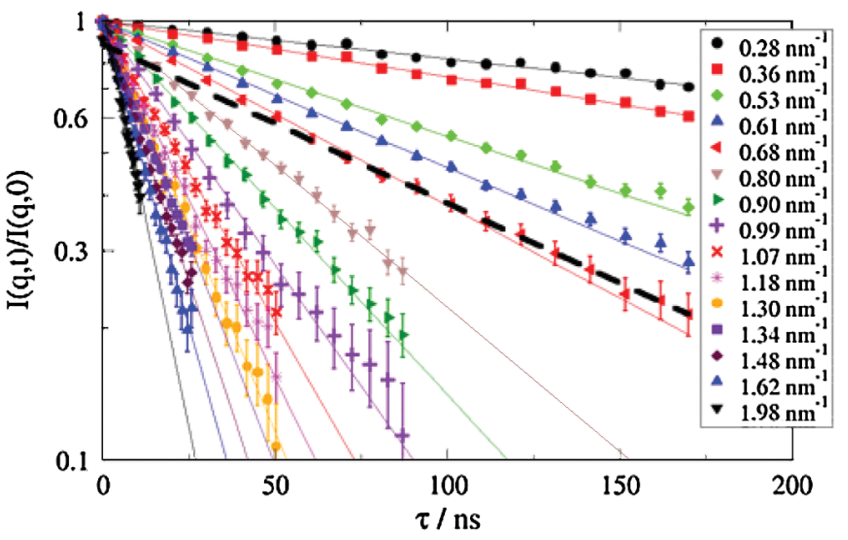

FIG. 2 (color). Intermediate scattering function measured by NSE for a protein concentration of $5 \% \mathrm{wt} / \mathrm{vol}$ with the bound cofactor. The broken line is a fit to $q=0.68 \mathrm{~nm}^{-1}$ for times above 75 ns.

$\partial /\left.\partial t \ln (I(q, t))\right|_{t=0}=-\Gamma(q)=-q^{2} D_{\text {eff }}(q) \quad$ were extracted with the relaxation rate $\Gamma(q)$ and the effective diffusion coefficient $D_{\text {eff }}(q)$ carrying the essential information on the observed dynamics. Comparing with the SANS data we note that NSE at small $q$ accesses the regime of intermolecular interactions, while at high $q$ it is sensitive to intramolecular phenomena.

At low concentration $D_{\text {eff }}(q)$ directly informs on the single particle motion. However, the necessity for high accuracy statistics requires also the evaluation of higher concentration data. In the range where the $S(q)$ is important, corrections for the repulsion between the tetramers and for the hydrodynamic interactions are necessary [8,9] $D_{\text {eff }}(q)=D_{\text {eff }}^{0} H(q) / S(q) ; D_{\text {eff }}^{0}(q)$ is the single tetramer effective diffusion coefficient and $H(q)$ the hydrodynamic function. An estimation for a spherical system shows that $H(q)$ has a similar shape as $S(q)$ and leads to an overall constant downshift of the high $q$ data [8,9]. Importantly, for $q>0.6 \mathrm{~nm}^{-1}$ any oscillations of $H(q)$ are below $1 \%$ and can be safely neglected. Therefore, we correct the data by the experimental $S(q)$ values and rescale the whole data sets with the ratio of the low $q$ translational diffusion coefficients $(1 / 0.84)$.

Figure 3(a) presents the resulting effective single tetramer diffusion coefficient $D_{\text {eff }}^{0}(q)$. The experimental data from the different concentrations collapse to one master curve showing a strong $q$ modulation with a maximum around $q=1 \mathrm{~nm}^{-1}$. Since $H(q)$ is independent of $q$ above $q=0.6 \mathrm{~nm}^{-1}$ the entire observed high $q$ modulation is determined by intra tetramer effects.

For the investigated $\mathrm{ADH}$ we found that the prime reason for the $q$ modulation of $D_{\text {eff }}^{0}(q)$ relates to the rotational diffusion of the aspherical tetramer. Including hydrodynamic interactions the first cumulant $D_{0}(q)$ originating from translational and rotational diffusion of a rigid protein may be precisely calculated with a $6 \times 6$ diffusion coefficient matrix $\mathbf{D}$, delivered by the computer code HYDROPRO 


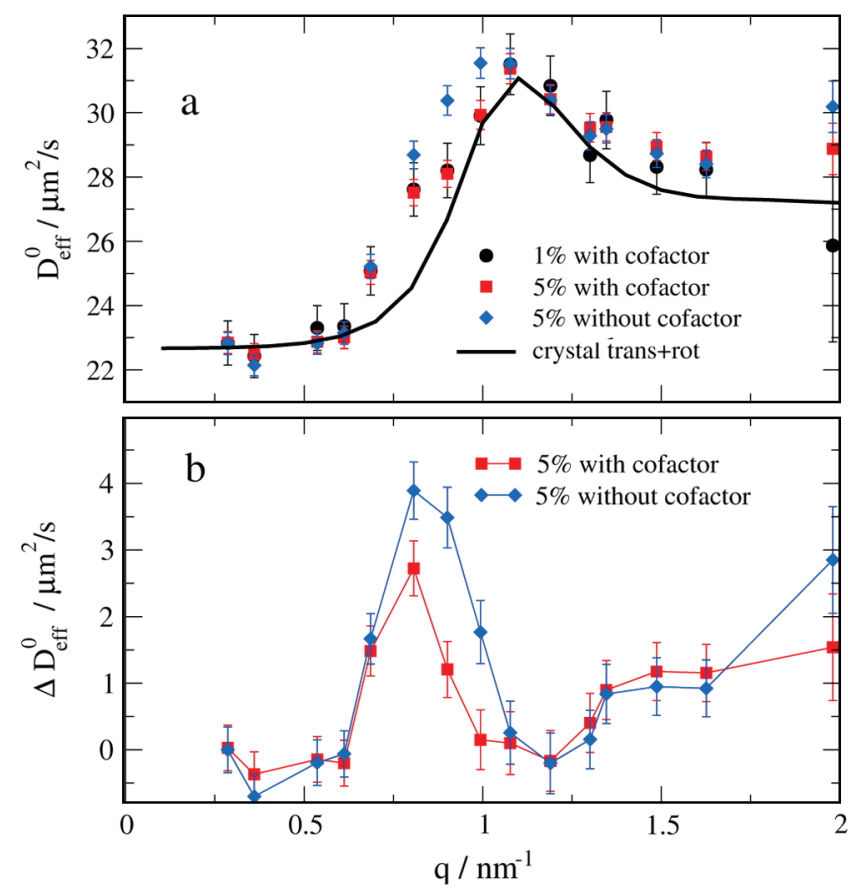

FIG. 3 (color). (a) Single tetramer diffusion coefficient $D_{\text {eff }}^{0}(q)$ after corrections with and without the bound cofactor. The black solid line represents the calculated effective diffusion coefficients for the $\mathrm{ADH}$ crystal structure, including translational and rotational diffusion. (b) Difference of the corrected diffusion coefficients and the calculated translational or rotational diffusion coefficient.

[10] with

$$
\begin{aligned}
D_{0}(q)= & \frac{1}{q^{2} F(q)} \sum_{j, k}\left\langle b_{j} \exp \left(-i \mathbf{q} \mathbf{r}_{\mathbf{j}}\right)\right. \\
& \left.\cdot\left(\underset{\mathbf{q} \times \mathbf{r}_{j}}{\mathbf{q}}\right) \mathbf{D}\left(\underset{\mathbf{q} \times \mathbf{r}_{k}}{\mathbf{q}}\right) b_{k} \exp \left(-i \mathbf{q} \mathbf{r}_{\mathbf{k}}\right)\right\rangle
\end{aligned}
$$

The sum runs over all subunits. The solid line in Fig. 3(a) presents the result $\left(D_{\text {trans }}=22.6 \mu \mathrm{m}^{2} / \mathrm{s} ; \quad D_{\text {rot }}=\right.$ $7.9 \times 10^{5} \mathrm{~s}^{-1}$ ). We note the excellent agreement with the experimental $D_{\text {trans }}$. The rise in $D_{\text {eff }}^{0}(q)$ largely stems from rotational diffusion. While the overall shape of $D_{\text {eff }}^{0}(q)$ is reasonably described, significant deviations are evident at the low $q$ flank. The assumption of a significant expansion of the protein structure $(\approx 15 \%)$ would shift the flank to match the position of the data points, but also decrease $D_{\text {eff }}^{0}(q)$ by a factor of 0.89 and rescale the SANS form factor by a factor $1 / 1.15$ to lower $q$ [Fig. 1(a) dashed line], all contradicting the experimental results. Thus, the deviations indicate additional dynamics not described by the rigid body translational and rotational diffusion. The deviations are shown in Fig. 3(b) with a pronounced peak around $0.8 \mathrm{~nm}^{-1}$. In the case of the bound cofactor the peak weakens and looses its high $q$ part.

In order to understand to a first approximation the collective motions underlying the dynamics displayed in
Fig. 3(b), we perform a normal mode [11] (NM) analysis based on the known tetrameric solution structure. Since we are interested in the softest large scale motions, elastic network models may be applied [12]. In this approach two atoms are connected by an elastic spring, if the equilibrium distance between them is sufficiently small. Close to the equilibrium the equations of motions can be linearized and the motions are described by a sum of independent oscillating normal modes with eigenvalues $\omega_{\alpha}^{2}$ and eigenvectors $\mathbf{e}_{i}^{\alpha}$. The first six trivial eigenmodes correspond to translation and rotation. The large scale slow motions, we are interested in, are overdamped modes. The oscillating part needs to be replaced by an exponential $\left(e^{-\lambda_{\alpha} t}\right)$ where the mode dependent relaxation rates $\lambda_{\alpha}$ contain the unknown friction factors within the molecule and with the surrounding water molecules.

With the structural information at hand using the web server ElNemo [13], the NM eigenvectors $\mathbf{e}_{\alpha}$ and eigenvalues $\omega_{\alpha}^{2}$ of the corresponding dynamical matrix are obtained. Thereby $\omega_{\alpha}^{2}$ is given relative to the lowest nontrivial mode. We note that the NM carry the structural information on the motional pattern of the underlying collective motion.

A first approximation for the dynamic form factor may be obtained in analogy to a phonon approximation of the cross section, which in fact is an expansion of the cross section with respect to small displacements.

$$
\begin{aligned}
F(q, t) \propto & F(q)+\sum_{\alpha} k_{\alpha} e^{-\lambda_{\alpha} t}\left\langle\sum_{k, l} b_{k} b_{l} \exp \left(i \mathbf{q}\left(\mathbf{r}_{k}-\mathbf{r}_{l}\right)\right)\right. \\
& \left.\cdot\left(\mathbf{q} \cdot \mathbf{e}_{k}^{\alpha}\right)\left(\mathbf{q} \cdot \mathbf{e}_{l}^{\alpha}\right)\right\rangle,
\end{aligned}
$$

where $k_{\alpha}=k_{B} T / \bar{m} \omega_{\alpha}^{2}$ is the mode dependent amplitude with average mass $\bar{m}$. In first cumulant approximation we have to take the logarithmic derivative with respect to time at time equal zero and finally obtain for the additional contribution of a normal mode $\alpha$

$$
\begin{aligned}
\Delta D_{\mathrm{eff}}^{\alpha}(q)= & \frac{\lambda_{\alpha} k_{\alpha}}{q^{2} F(q)} \\
& \cdot\left\langle\sum_{k, l} b_{k} b_{l} \exp \left(i \mathbf{q}\left(\mathbf{r}_{k}-\mathbf{r}_{l}\right)\right)\left(\mathbf{q} \cdot \mathbf{e}_{k}^{\alpha}\right)\left(\mathbf{q} \cdot \mathbf{e}_{l}^{\alpha}\right)\right\rangle .
\end{aligned}
$$

An evaluation of the mode form factors $\Delta D_{\text {eff }}^{\alpha}(q) / \lambda_{\alpha}$ shows that the most prominent contributions to $\Delta D_{\text {eff }}^{0}(q)$ at low $q$ arise from the first nontrivial mode 7 and mode 11, which are displayed in Fig. 4(a) with and without the cofactor.

Figures 4(b) and 4(c) display the corresponding motional patterns. We note the astonishingly close resemblance of the predicted $\Delta D_{\text {eff }}(q)$ with the observation displayed in Fig. 3(b), though the experimental $q$ range is somewhat lower than that calculated.

Concerning molecular function, mode 7 is of highest interest; here the outside catalytic domains move with 


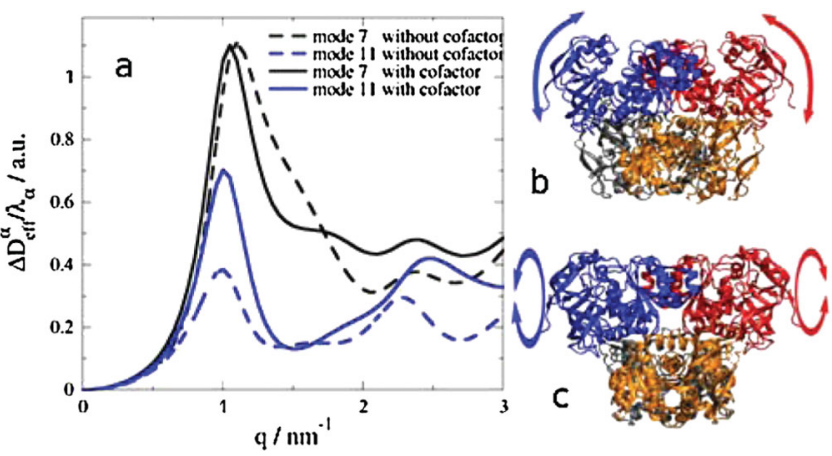

FIG. 4 (color). (a) Diffusion form factor of the normal modes 7 and 11 for the protein configuration with and without the cofactor. (b) Motional pattern of mode 7: Without cofactor the exterior domain (catalytic domain) tilts outwards and opens the cleft. The inner domain with connection points between the monomers remains stiff. (c) Motional pattern of mode 11: With and without the bound cofactor the monomers within a dimer exhibit torsional motion around the long dimer axis (in the image plane), which is more pronounced with the cofactor.

respect to the relatively rigid tetrameric core part, thereby changing the width of the cleft $[14,15]$. With the cofactor the mode 7 form factor looses its high $q$ flank as observed in the experiment and the vibrational pattern indicates, though similar, a less flexible cleft region. While the original crystal structure only displays a small narrow pocket, where the cofactor has to fit in, the opening motion of mode 7 provides more configurational freedom improving the ability to accept or release the cofactor for biological function. The other contributing mode (11) also shows a pronounced low $q$ peak. It is of more mechanistic nature describing the torsion of the dimer-the cleft is not opened.

The small mismatch in $q$ scales may result from anharmonic effects affecting mainly the outer parts of the molecule or from inhomogenously distributed friction reducing the motion of the inner residues. Both would enhance the motional amplitudes of the outer atoms shifting thereby the dynamic features to lower $q$.

Finally, we estimate amplitude and force constant for the modes relevant for the low $q$ dynamic feature in reevaluating NSE curves at $q=0.68 \mathrm{~nm}^{-1}$. (Figure 2). Describing the long time part $(t>75 \mathrm{~ns})$ by the time constant prediction for combined translational rotational diffusion at 5\% conc. ( $\tau=112 \mathrm{~ns}$ ) and extrapolating to $t=0$ we find an amplitude of about 0.9. The additional short time part $(t<$ $75 \mathrm{~ns}$ ) decays with a time constant of $\tau \approx 30 \mathrm{~ns}$, much smaller than time constants for translation ( $\tau \approx 100 \mathrm{~ns}$ ) or rotation $(\tau>100 \mathrm{~ns})$ which are here not distinguishable experimentally. Via a Debye-Waller factor estimate we get the relevant mean square displacement of the internal mode as $-q^{2}\left\langle r^{2}\right\rangle / 3=\ln 0.9$ revealing $\left\langle r^{2}\right\rangle=0.7 \mathrm{~nm}^{2}$. From equipartition we then may obtain the spring constant $f=k_{B} T /\left\langle r^{2}\right\rangle=5.6 \mathrm{pN} / \mathrm{nm}$-a value well in the range of spring constants considered for bio molecules $[16,17]$. We note that the NM configurational fluctuations with this amplitude are consistent with the observed SANS data.

In summary, we have studied the internal dynamics of tetrameric $\mathrm{ADH}$ on the space time scale of the protein. The revealed large scale correlated domain motions in term of a NM analysis are understood as a result of the moving exterior catalytic domains relative to the stiff core of the tetrameric protein. The motional amplitude of about $0.8 \mathrm{~nm}$ is significant in order to assist the functionally necessary incorporation of the cofactor.

We thank S. Born for protein sample preparation and ADH activity measurements. This research project was supported by the EU Commission under the 6th Framework Program: Strengthening the European Research Infrastructures.

[1] F. Colonna-Cesari et al., J. Biol. Chem. 261, 15273 (1986).

[2] S. Ramaswamy et al., J. Mol. Biol. 235, 777 (1994).

[3] E. Negelein and H.J. Wulff, Biochemische Zeitschrift 293, 351 (1937).

[4] S. Hammes-Schiffer and S. J. Benkovic, Annu. Rev. Biochem. 75, 519 (2006).

[5] J. H. R. Kägi and B. L. Vallee, J. Biol. Chem. 235, 3188 (1960).

[6] B. Jacrot, Rep. Prog. Phys. 39, 911 (1976).

[7] K. Hinsen, J. Comput. Chem. 21, 79 (2000).

[8] J. Gapinski et al., J. Chem. Phys. 123, 054708 (2005).

[9] J. Gapinski et al., J. Chem. Phys. 126, 104905 (2007).

[10] J. G. de la Torre, M. L. Huertas, and B. Carrasco, Biophys. J. 78, 719 (2000).

[11] F. Tama and Y. H. Sanejouand, Protein Eng. 14, 1 (2001).

[12] M. M. Tirion, Phys. Rev. Lett. 77, 1905 (1996).

[13] K. Suhre and Y.H. Sanejouand, Nucleic Acids Res. 32, W610 (2004).

[14] J. Luo and T. C. Bruice, Proc. Natl. Acad. Sci. U.S.A. 99, 16597 (2002).

[15] J. Luo and T. C. Bruice, Biophys. Chem. 126, 80 (2007).

[16] B. T. Marshall et al., Biophys. J. 90, 681 (2006).

[17] L. A. Chtcheglova et al., Biophys. J. 86, 1177 (2004). 